\title{
Adopting the O'Driscoll regime may optimize caesarian section rate in the primiparous woman.
}

\author{
Peter Petros ${ }^{1}$ \\ ${ }^{1}$ University of Western Australia
}

May 19, 2021

Professor PEP Petros DSc DS (UWA) PhD (Uppsala) MB BS MD (Syd) FRCOG (Lond)

31/93 Elizabeth Bay Rd, Elizabeth Bay NSW 2011 AUSTRALIA

Reconstructive Pelvic Floor Surgeon and Certified Urogynaecologist (retired)

Formerly University of NSW Professorial Dept of Surgery, St Vincent's Hospital Sydney (retired)

Adjunct Professor, University of Western Australia School of Mechanical and Chemical Engineering, Perth WA (current)

Tel 61293613853 Cellphone (AUST) 61411181731

Email pp@kvinno.com website www.integraltheory.org

Re Global rising rates of caesarean sections

Patrick Chien

First published: 05 March 2021

https://doi.org/10.1111/1471-0528.166

No of words 441

Conflicts None

Funding NIL

The Editors

BJOG

Running head O'Driscoll regime may optimize CS rate

Adopting the O'Driscoll regime may optimize caesarian section rate in the primiparous woman.

Dr Chen asks, "What is the optimum rate of caesarean section delivery? This is a question that has bedevilled many obstetricians over the last half century."[ 1] We believe our recently published OASIS study may shed some light on this important question, albeit from a different angle. We examined the effect of passive management of labour on OASIS [2] and found that the O'Driscoll active management method vastly reduced OASIS in primiparas without increase in caesarian section (CS). Routine adoption of this method may go a long way towards answering Dr Chen's question. The genius of the O'Driscoll method is that it is a "zero sum" * protocol which is tailors CS to an individual patient. It automatically takes into account all three "Ps", powers, passengers, passages, irrespective of which one is causing the problem. 


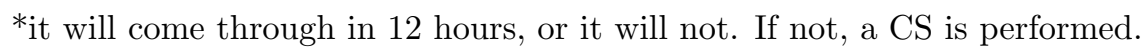

We carried out a comparative study of third and fourth degree anal sphincter tears in all primiparas delivering at term in the years 2010-2015 in six Sydney public teaching hospitals, which were under MANDATORY direction from the Director General of NSW Health for labour to proceed without augmentation. The study comprised 130,000 women. We compared teaching hospital data with data from patients delivered in six adjacent Sydney private hospitals which used augmented labour. A second comparator was from the National Women's Hospital, Dublin, where the O'Driscoll active management is performed under midwife direction. The mean third and fourth degree anal sphincter tear rate was $8.17 \%$ for NSW public teaching hospitals, $1.52 \%$ for the adjacent private hospitals in the same period $(\mathrm{p}<0.0003)$ and $2.6 \%$ for NWH Dublin. The emergency CS rate between 2010-2015 was $13.7 \%$ private, $12.7 \%$ public, $7.9 \%$ NHW Dublin. However, there was an increase in rate of epidurals, forceps/ventouse and lower Apgar scores in NSW public hospitals.

We attributed the rise in OASI to poor contractions causing deflexion of the head, increasing the cephalic diameter from $9.5 \mathrm{~cm}$ to $11.2 \mathrm{~cm}$ with consequent increased stress on the sphincters.

In an accompanying editorial [3], we examined the longer-term damaging effect of a larger diameter head pushing through the pelvis on the ligaments, tissues and muscles with regard to future prolapse, bladder, bowel symptoms. In this context, we described OASIS as "the canary in the coalmine", a harbinger of such problems in the future for the OASIS women.

It would seem that the O'Driscoll active management regime, which limits labour to 12 hours without increasing the caesarean section rate, may not only protect from OASIS, POP and incontinence, but also, provide an optimum caesarian section rate for the individual woman.

\section{References}

Patrick Chien Global rising rates of caesarean sections BJOG

First published: 05 March 2021 https://doi.org/10.1111/1471-0528.166

Beale RM Petros PE Passive management of labour may predispose to anal

sphincter injury Int Urogynecol J. 2020 Sep;31(9):1943-1947. doi: 10.1007/s00192-019-04183-6. Epub 2020 $\operatorname{Jan} 7$.

Peter Petros , Mark Richard Beale , Burghard Abendstein ... Editorial

The mechanics and biomechanics of OASIS POP and incontinence-is active management of labour protective? ... management of labour protective? Int Urogynecol J. 2020 Sep;31(9):1727-1728. doi: 10.1007/s00192020-04279-4. 